\title{
Variable sex ratio among colonies of ants
}

\author{
Steven A. Frank \\ Department of Biology, University of Michigan, Ann Arbor, MI 48109-1048, USA \\ Received June 18, 1986 / Accepted October 26, 1986
}

\begin{abstract}
Summary. Small colonies of ants often produce mostly male alates, while large colonies produce mostly female alates. I present a simple model consistent with this pattern in which males that compete for mates are related (Local Mate Competition). The model explains the observed trend even when relatedness among competing males is low, so that there is only a negligible effect on the predicted sex allocation ratio in the population. The reverse trend is expected when there is competition among related females for a limited resource, such as nest sites (Local Resource Competition); small broods are predicted to be mostly female and large broods are predicted to be mostly male.
\end{abstract}

\section{Introduction}

There is a tendency in many ant species for small colonies to produce mostly male alates, and for large colonies to produce mostly female alates (data reviewed by Nonacs 1986). Two causal explanations have been proposed. First, Herbers (1984) found that in Leptothorax longispinosus the investment ratio in males declined as the number of workers in the colony increased. She suggested that as worker numbers rise, workers increasingly gain control from the queen(s) over the colony allocation ratio. Because of the haplodiploid genetic system of ants, workers favor a female biased allocation ratio, since they are more closely related to female reproductives than to male reproductives, while queens are equally related to male and female progeny, and so favor equal allocation in the sexes (Trivers and Hare 1976). Second, Nonacs (1986) suggested that when resources are scarce, females are channeled into becoming workers rather than alates, while males still develop into alates, and that when resources are abundant, both males and females develop into alates. The data Nonacs (1986) analyzed show a general trend of an increasing ratio of female to male alates as the total number of alates increases, which is consistent with his hypothesis.

I propose an alternate hypothesis which is also generally consistent with the observed trends. If there is any competition among related males for access to mates (Local Mate Competition - LMC, Hamilton 1967), then each colony is predicted to produce all males up to some threshold, and then if resources permit, to augment this threshold value for males by producing all females (Frank 1985, 1987; Yamaguchi 1985). I call this the Constant Male Hypothesis (CMH). This prediction holds even if the amount of LMC is small, so that the effect on the population allocation ratio is negligible. A corollary of this hypothesis is that if competition among related females for nesting sites or other resources (Local Resource Competition LRC, Clark 1978) is more intense than LMC, then small colonies are predicted to produce mostly females, and when possible, to augment initial production of females with male alates. This second prediction also holds when the intensities of both LMC and LRC are small, so that the effect on the population allocation ratio may not be measurable. Support of this hypothesis does not necessarily have any effect on deciding between a workerqueen conflict interpretation of the population sex allocation ratio (Trivers and Hare 1976) and a LMC interpretation (Alexander and Sherman 1977).

A general method for analyzing the effects of LMC, LRC, polygyny, multiple mating, and worker or queen control, is presented in the Appendix, which will be useful when testing hypotheses about sex allocation in ants. 


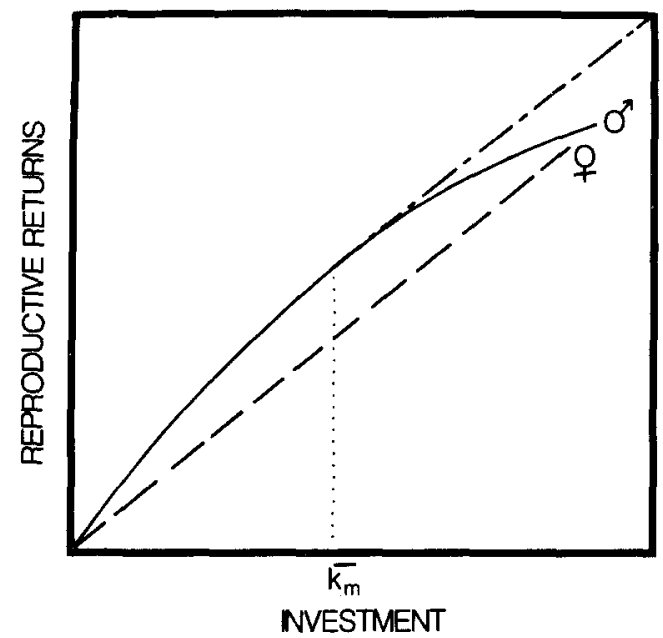

Fig. 1. Reproductive returns on males and females as a function of investment, under weak Local Mate Competition. Up to $k_{m}$ the rate of returns on male investment is greater than on female investment, so colonies with $\overline{k_{m}}$ resources are expected to produce all males. After $k_{m}$ the rate of returns on female investment is greater than on male investment, so resources in excess of $\overline{k_{m}}$ are allocated to females, yielding total reproductive returns for the brood as shown in the dot-dash line

\section{The constant male hypothesis}

Frank (1985) and Yamaguchi (1985) showed that if there is LMC and variation in amount of resources that individuals (colonies) have to invest in offspring, then at equilibrium all individuals will invest the same amount of resources in males, and will use all remaining resources for making females. Colonies with insufficient resources to make the minimum male investment will also make all males (Yamaguchi 1985). Frank (1987) derived this same result for the general case in which the reproductive returns on investment in females are linear, and there are diminishing returns on investment in males. LMC is a special case of this general result, since returns for females will increase linearly as the number of females produced increases (in the absence of competition among female relatives, see below), and inclusive fitness returns for male investment will increase at a diminishing rate as the number of males produced increases, since there will be increasing competition among male relatives. Note that the LMC argument does not require that there be any inbreeding (Frank 1986a), nor does it require that there actually be much competition among male relatives. It does require that if the number of males produced by a colony were to increase, then competition among male relatives would also increase.

The logic of the $\mathrm{CMH}$ is shown in Fig. 1 (see Frank 1987, for further details). The rate of returns

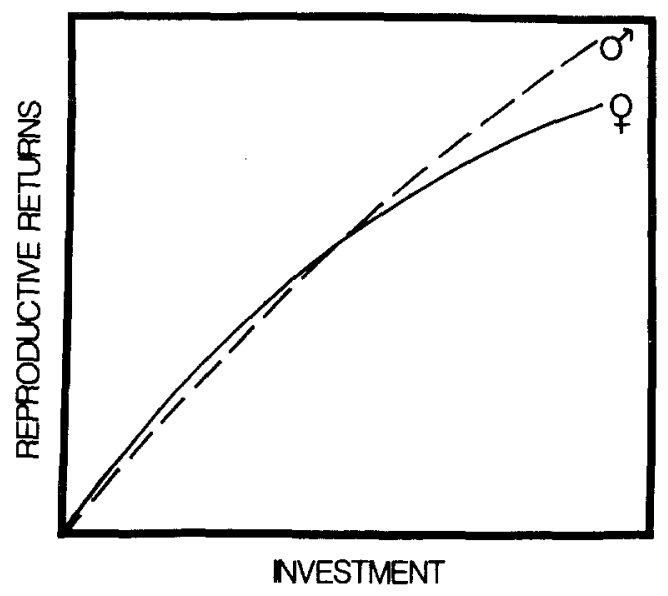

Fig. 2. Reproductive returns on investment in males and females, when there is both weak Local Mate Competition (LMC) among males and relatively more intense Loeal Resource Competition (LRC) among females. Because LRC is more intense than LMC in this example, the rate of returns on female investment declines more rapidly than does the rate of returns on male investment. Colonies with small amounts of energy to invest are predicted to produce mostly females, and those with large broods are expected to produce mostly males. The rate at which a colony is expected to produce increasingly more males as investment increases depends on the relative curvature of the male and female return curves - e.g., on the ratio of the second derivatives for smooth curves

on male investment is greater than the rate for female investment up to an investment of $\overline{k_{m}}$ resource units, so producing all males is favored for colonies with less than $\overline{k_{m}}$ resources. After $\overline{k_{m}}$ the rate of returns on female investment exceeds the rate on male investment, so the remainder of the colony's energy will be spent most profitably on females.

If there were competition among female relatives (LRC) and no competition among male relatives (LMC), then this situation would be described by switching the male and female labels on the curves in Fig. 1. Females would be favored in small colonies, with an increasing proportion of males produced in large colonies. The argument is similar if there is both LMC and LRC (Fig. 2). Small colonies will produce the sex that has the most intense competition, and therefore, the faster rate at which returns diminish with increasing investment. Once again, these effects can occur even if the intensities of both LMC and LRC are small.

\section{Population sex allocation for haplodiploidy}

An interesting result of the Constant Male Hypothesis is that if the smallest colony has at least $\overline{k_{m}}$ resources to invest in reproduction (see Fig. 1), 
then the population allocation ratio is independent of the variation in reproductive output among colonies (Frank 1985, 1987, Yamaguchi 1985). A general result for the population allocation ratio can therefore be obtained by assuming that all colonies have the same amount of resources available for reproduction. The observed population allocation ratio can then be used to obtain an estimate of the intensity of LMC that is consistent with the data. A useful prediction for social insects must take into account both haplodiploidy and the possibility of either queen or worker control of the allocation ratio. Previous authors have given predictions for special cases (e.g., Uyenoyama and Bengtsson 1981, 1982). In the Appendix, present a more general result, which also clarifies the causal mechanisms underlying sex allocation biases (see also Taylor 1987).

\section{Predictions of the constant male hypothesis}

In this section I will present a method for estimating the amount of LMC that one must invoke in order to explain observable variation in sex allocation among colonies (Yamaguchi 1985 took a similar approach for the diploid aphids that she studied). Although the method is simple and straightforward, a strict interpretation requires certain patterns in the data that are not met by the information currently available (discussed below). There are two main reasons for pursuing the theory in a somewhat formal manner. (i) The theory will show that a very small amount of LMC is sufficient to explain why much variation among colonies might exist. (ii) A formal approach will show how the assumptions may be modified realistically in order to give results more consistent with the data. This is a way of fitting theory to the data, which is useful when searching for a plausible explanation for a widely observed pattern.

First we need some notation. Let $k_{m}(j)$ and $k_{f}(j)$ be the amount of resources invested by the $j^{\text {th }}$ colony in males and females, respectively; define $N_{m}(j)$ and $N_{f}(j)$, as the number of males and females, and $W_{m}(j)$ and $W_{f}(j)$ as the weight per male and per female. Then

$k_{m}(j)=N_{m}(j)\left[\beta+W_{m}(j)\right]$

$k_{f}(j)=N_{f}(j)\left[\beta+W_{f}(j)\right]$,

where $\beta$ is the initial investment per individual offspring not reflected in its final weight. Usually $\beta$ is assumed to be zero, and weights alone are used to estimate investment. It seems unlikely that $\beta$ is actually zero, but since no information is pre-

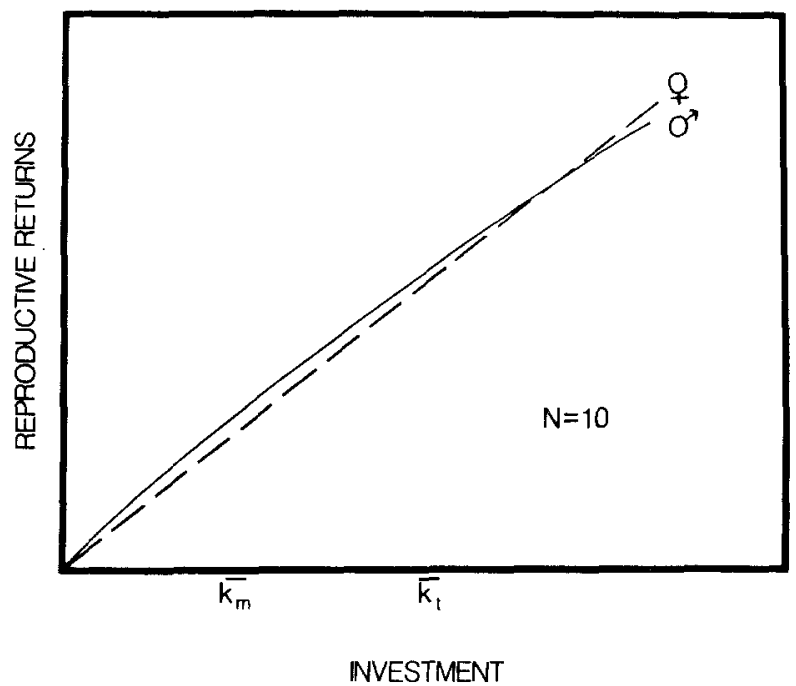

Fig. 3. The reproductive returns on male and female investment when the number of colonies contributing to a mating swarm is ten, there is no Local Resource Competition, and the population is near equilibrium. The average investment per colony is $\overline{k_{t}}$, and investment in males by each colony is $\overline{k_{m}}$

sently available, I will also assume that $\beta$ is zero. However, it is worthwhile to keep in mind the full representation for investment shown above.

The Constant Male Hypothesis suggests that if $k_{t}(j)=k_{m}(j)+k_{f}(j)$ is such that $k_{t}(j) \geq \overline{k_{m}}$ for all colonies $j$, then $k_{m}(j)=\overline{k_{m}}$ for all $j$, where overbars denote averages over $j$ (Frank 1985, 1987; Yamaguchi 1985). In words, if every colony has at least enough energy, $k_{t}(j)$, to invest at a level equal to the average male investment in the population, $\overline{k_{m}}$, then each colony will invest in males exactly the population average, $\overline{k_{m}}$. So, if the total energy for a colony, $k_{t}(j)$, is just equal to $\overline{k_{m}}$, then that colony will make only males, and if $k_{t}(j)$ exceeds $\overline{k_{m}}$, then the colony will invest $\overline{k_{m}}$ in males and $k_{t}(j)$ $-\overline{k_{m}}$ in females.

The predicted population allocation ratio $x^{*}$ is given in Eqs. (A.2, A.3) of the Appendix. An estimate for the population allocation ratio can be obtained by estimating $\overline{k_{m}} / \overline{k_{t}}$. Call this estimate $\hat{x}$. If we assume that relatedness among neighboring monogynous colonies is low, then the predicted population allocation ratio $x^{*}$ depends only on $N$, the number of colonies contributing alates to a local swarm. Given an estimate for the population allocation ratio, $\hat{x}$, one can solve for an estimate of the number of colonies contributing to the swarm (see Appendix):

(queen control) $\quad \hat{N}=\frac{(3-\hat{x})+\sqrt{\hat{x}^{2}+10 \hat{x}+1}}{4(1-2 \hat{x})}$ 
(worker control) $\hat{N}=\frac{1-\hat{x}}{1-4 \hat{x}}$.

Consider two examples that illustrate how sex ratio variation among colonies could be explained. First, suppose that colonies in the hypothetical population we are observing produce approximately the same number of males, but vary in the production of females. This is consistent with the prediction of the CMH. Now, suppose that our estimate for the population allocation ratio is $\hat{x}=$ 0.45 , and we are willing to assume queen control in this case. Then $\hat{N}=10$ is a reasonable estimate for the number of colonies contributing to the local mating swarm that would be consistent with the prediction of the $\mathrm{CMH}$, according to Eq. (1). Similarly, if $\hat{x}$ is 0.47 , then $\hat{N}=20$ is a reasonable estimate for the number of interbreeding colonies in each patch. Under worker control, Eq. (2), $\hat{x}=0.23$ suggests $\hat{N}=10$, and $\hat{x}=0.24$ implies $\hat{N}=19$.

Figure 3 shows the shapes and relative positions of the returns on male and female investment for $N=10$, which are very nearly the same for both queen and worker control. This assumes that the population is in equilibrium and that the smallest colony has resources at least equal to $x^{*} \overline{k_{t}}=\overline{k_{m}}$. Yamaguchi (1985) has shown that when the smallest colonies (individuals) have less that this threshold amount, then small colonies are still predicted to produce all males, but there is a small shift in the population allocation ratio and the threshold value. The qualitative trends are unchanged. The similar shapes of the male and female curves in Fig. 3 suggest that the approach to equilibrium will be slow, so that even when the postulated forces are occurring, one would not necessarily expect the observed patterns to match neatly with the predicted patterns. This is discussed further when considering some of the available data.

The most interesting conclusion about variation among colonies from the Constant Male $\mathrm{Hy}$ pothesis is that a small amount of LMC can explain why small colonies produce all males, and larger colonies produce mostly females. Up to the threshold point $\overline{k_{m}}=\overline{k_{t}} x^{*}$, the returns on male investment are greater than the returns on female investment, and after the threshold the returns on female investment are greater (Figs. 1, 3). An interesting corollary of the $\mathrm{CMH}$ is that if competition among female relatives for resources such as nest sites (local resource competition - LRC, see Clark 1978 ) is more intense than competition among related males for access to mates (LMC), then the reverse trend is predicted: small colonies will produce mostly females and large colonies will pro- duce mostly males. Note that the intensities of both LMC and LRC may be weak, but still lead to this prediction (Fig. 2).

\section{Discussion of alternate hypotheses}

Three causal explanations have been proposed to account for the observed association between colony size and sex allocation. Here I briefly look at observed qualitative trends in ants and other social insects with respect to these three hypotheses.

(i) Herbers (1984) found that in Leptothorax longispinosus the investment ratio in males declined as the number of workers in the colony increased. She interpretated this as the workers increasingly winning the conflict over the allocation ratio as the colony grows larger. Nonacs (1986) pointed out that this trend was found in both monogynous and polygynous colonies in Herbers' study, which he suggested is inconsistent with her explanation, since worker and queen optima converge as the number of queens increases. However, this convergence of optima depends on particular assumptions which may not always be met, and deserves further study. For example, if there were many unrelated queens, and workers could not distinguish between kin and non-kin, then workers would favor a 1:3 investment in their sibs, and in a large population would be indifferent towards non-kin. Since they may not be able to distinguish between kin and non-kin, they may still favor a $1: 3$ ratio (see Appendix). Herbers' hypothesis therefore remains a plausible explanation.

(ii) Nonacs (1986) proposed that both male and female eggs are laid, and that when resources are scarce the female eggs are raised as workers. This would explain the observed colony level pattern; however, the logic underlying this hypothesis is not particularly compelling. If resources are predictably scarce at the time the eggs are laid, then a division among investment in workers, gynes and males can be made most efficiently at this time. If resources become unpredictably scarce while raising the brood, no obvious benefit accrues to switching some or all would-be gynes into workers.

(iii) The Constant Male Hypothesis presented above suggests that when there is any Local Mate Competition, however slight, small colonies are favored to make mostly males, and large colonies are expected to increase their investment in females as their total brood increases. Three testable predictions follow from this hypothesis.

(a) Genetic analyses of mating swarms by electrophoresis could potentially detect small amounts 
of LMC. Under the $\mathrm{CMH}$, as the measured amount of LMC increases, the likelihood of specialization according to colony size increases. See the Appendix for a discussion of how to interpret mating swarm data.

(b) In a polygynous colony in which queens are not related, there may be competition among males from the same colony with only a small amount of LMC, since LMC depends on relatedness among competing males. In general, the amount of LMC (and competition among female reproductives, LRC) depends on the number of offspring per queen and the relatedness among queens (see Appendix). If in a particular polygynous species the queens are distantly related, then LMC is less likely and, under the $\mathrm{CMH}$, specialization is also less likely.

(c) If competition for nest sites and other resources is greater among females (LRC) than is competition among males for mates (LMC), then small broods are predicted to be female biased and large broods male biased (Fig. 2). Nest sites in the social wasp Polistes have been reported as relatively rare, and potentially a limited resource (Noonan 1979). Strassmann (1984) found that, in two of four years in her study, there was a significant negative association between brood size and proportion of males. She suggested that when the end of the season is unpredictable, females should be produced before males, since a female Polistes may become either a worker or gyne after it ecloses. Thus, if the season ended unusually early, recently born females would become reproductives, while if the season lasted longer, these females could stay and help rear another, much larger brood with both male and female reproductives. According to this idea, small colony size, early termination of breeding, and female-biased sex ratios would be positively associated. While these Polistes data are certainly not strong support of the LRC hypothesis (c), and Strassmann (1984) has proposed an alternative explanation, they do suggest the possibility that additional data on the relative intensities of LMC and LRC, with respect to colony specialization, will be interesting.

The CMH makes a very sharp prediction: that the number of males be constant. While there are insufficient data to test the above predictions, it is already clear that the constant male aspect of the hypothesis will not be strongly supported by the data (data and references in Nonacs 1986). This does not necessarily weaken the above predictions for three reasons. First, the constant male part of the hypothesis emerges only when each colony has perfect information about the intensities of LMC and LRC, or when these intensities have been sufficiently constant for a genetically determined phenotype to arise, and when the population is at equilibrium - which it certainly never is. Figure 3 shows that selection is weak near equilibrium, so that the forces maintaining constant male behavior are expected to be weak. Farther from equilibrium the forces affecting sex ratios will be stronger, so that the general tendencies (a) - (c) above are more likely than rigid constant male behavior by the population. Second, the weaker LMC becomes, the weaker the force favoring specialization. Consistent with this trend, Yamaguchi (1985) found that the data on aphids gave a reasonably close fit to the $\mathrm{CMH}$, and that the estimated amount of LMC was equivalent to four foundresses per patch, which is quite strong (i.e., $\hat{N}=4$, in the sense of Eq. 1). I suggest that in a population with weaker $\mathrm{LMC}$, the CMH trend would exist, but that the fit would not be as close. Third, when there is both LMC and LRC, the switch towards the production of females is less rapid. The rate of switching to female production is expected to depend on the relative intensities of LMC and LRC (Fig. 2).

One difficulty with the $\mathrm{CMH}$ is that it is consistent with a number of different patterns, with only moderate adjustments in the underlying assumptions. Support for this hypothesis will therefore be difficult in any particular case, but the hypothesis does make a number of strong predictions about general trends across species. An advantage of this generality is that it will provide a framework for organizing large amounts of information. For example, relatedness in mating swarms, number of queens and relatedness among queens in polygynous colonies, and competition among gynes for nest sites, all have a logical association with colony sex allocation ratio as a function of brood size.

Acknowledgements. Peter Smouse gave helpful comments on an earlier draft of the manuscript. This work was supported by the NIH National Research Service Award 1-T32-07544-07 and NIH-RO1-GM32589.

\section{Appendix}

Here I derive predictions for the population sex allocation ratio for haplodiploidy with Local Mate Competition (LMC) among males, Local Resource Competition (LRC) among gynes, polygyny, multiple mating, and worker or queen control. The results include any level of relatedness among queens, inbreeding, and relatedness among competing males or females, and are therefore helpful for understanding sex allocation in ants in general, and the Constant Male Hypothesis (CMH) in particular.

Taylor (1987) has recently developed a general method for problems when relatives interact, and presented the case of sex allocation in haplodiploids with monogynous queen control 
and single mating as an illustration. I rederive this and other results mentioned above by my own general "genetic value" method (Frank 1986a, b). My method is heuristic rather than mathematically formal as Taylor's, and has the advantage that it highlights causal mechanisms in a biologically intuitive way. This makes extensions based on complex natural histories easier to follow, and points to the sorts of data that must be collected and the difficulties that may be encountered when testing various hypotheses. Taylor's (1987) paper contains an excellent discussion of the assumptions and formal aspects of this sort of heuristic approach. See also the cautionary remarks in Frank $(1986 a, b, c)$.

By the genetic value approach, the equilibrium sex allocation ratio is equal to the rate at which alleles from the controlling genotype are transmitted to future generations in return for a unit of investment in males, relative to the rate per unit investment in females (Frank 1986a, b). With the usual simplifying assumptions, such as large population size, discrete generations at both the individual and colony level (see Taylor 1987), the ESS sex allocation ratio can be obtained by assuming that males and females are equally costly to produce, and then simply counting the number of alleles identical by descent (ibd) that will be transmitted to the grandprogeny generation by the production of an extra male, relative to the number transmitted by the production of an extra female. Some of the difficulties of measuring the transmission of ibd alleles are discussed by Pamilo and Crozier (1982) and Taylor (1987). The task for haplodiploids is easy when the life cycle is kept simple, such as only queens laying eggs, and discrete reproductive cycles. In this case, the reproductive values of alleles with respect to transmission to future generations (sensu Fisher 1958) are equal in males and females, and so a simple count is sufficient. The genetic values of males and females will in each case have a direct component, the number of alleles ibd passed directly through the individual to future generations, and will additionally have a component that depends on interactions among relatives.

Let $B_{c \rightarrow m}$ and $B_{c \rightarrow f}$ be the number of alleles ibd from the controlling genotype (queen or worker) in a male $(m)$ or female $(f)$ progeny - the direct component. With LMC among males, the total number of alleles transmitted by a male must be discounted by any alleles ibd that he interferes with while competing for mates. Let the average number of alleles ibd in a male's (haploid) competitors be $F_{c \rightarrow m \rightarrow \mu}$, the gametic correlation $F$ (Wright 1969) between the controlling genotype $c$, and the male progeny $m$ 's male competitors $(\mu)$. Thus the overall genetic value of a male is $B_{c \rightarrow m}-F_{c \rightarrow m \rightarrow \mu}$. The value of an extra female must be augmented by the number of alleles in her mate that are ibd to control, since the total number of ibd alleles transmitted through an extra daughter depends on both the daughter's and mate's genotypes, and on the genetic system. For haploid males this number is $F_{c \rightarrow f \rightarrow \mu}$, the gametic correlation between control $(c)$ and female progeny's $(f)$ mates $(\mu)$. Female progeny may also compete for limited resources, such as nest sites, with other females and their mates. The number of ibd alleles in diploid female competitors in $2 F_{c \rightarrow f \rightarrow \phi}$, and in female competitors' (haploid) mates, $F_{\mathrm{c} \rightarrow f \rightarrow \phi \rightarrow \mu}$, where it is assumed that only competitors for limited resources are included among the female group $(\phi)$ (see below).

The ESS sex allocation ratio is the genetic value of males relative to the genetic value of females, or

$B_{c \rightarrow m}-F_{c \rightarrow m \rightarrow \mu}: B_{\mathrm{c} \rightarrow f}+F_{c \rightarrow f \rightarrow \mu}-\left(2 F_{c \rightarrow f \rightarrow \phi}+F_{c \rightarrow f \rightarrow \phi \rightarrow \mu}\right)$

The form of Eq. (A.1) is useful for identifying causal mechanisms underlying sex allocation biases, but is difficult to apply. To obtain a more applicable form, define $F_{\phi \mu}, F_{\mu \mu}$, and $F_{\phi \phi}$ as the gametic correlations among individuals in the mating swarm between, respectively, males and females (the usual inbreeding coefficient), males and males, and females and females. There are two cases to consider, queen control $(c=q)$ and worker control $(c=w)$. First, the value of the $B$ 's:

$$
\begin{aligned}
B_{q \rightarrow m}= & (1 / 2)\left(1+F_{\phi \mu}\right) \\
B_{q \rightarrow f}= & (1 / 2)\left(1+3 F_{\phi \mu}\right) \\
B_{w \rightarrow m}= & p(1 / 4)\left(1+3 F_{\phi \mu}\right)+(1-p)(1 / 2)\left(F_{q q}+F_{q q_{\mu}}\right) \\
B_{w \rightarrow f}= & p(1 / 4)\left(1+2 F_{q \mu \mu}+5 F_{\phi \mu}\right) \\
& +(1-p)(1 / 2)\left(F_{q q}+2 F_{q q_{i j}}+F_{q_{i q} q_{\mu}}\right)
\end{aligned}
$$

where $p$ is the expected proportion of reproductives that have the same mother as a worker, $F_{q q}$ is the correlation among gametes of different queens from the same colony, $F_{q q_{\mu}}$ is the correlation among gametes from a queen and the mates of other queens in the same colony, $F_{q_{\mu \mu}}$ is the correlation among gametes of the mates of a single queen (which is one for single mating), and $F_{q_{\mu} q_{\mu}}$ is the correlation among gametes of mates from different queens in the same colony. With estimates of $F_{\phi \mu}, F_{\phi \phi}$, and $F_{\mu \mu}$ from the mating swarm, and assumptions or estimates for number of matings and how queens settle together to form polygynous colonies, all four $B$ 's can be estimated. Single mating or monogyny are of course special cases of these more general expressions.

It is sometimes suggested that under worker control and polygyny, the workers favor an allocation ratio that approaches $1: 1$ as the number of queens increases (Herbers 1984; Nonacs 1986). An example shows that this trend is unlikely, and that worker controlled ratios should be nearly independent of queen number, excluding any effect that may be caused by LMC, and eggs laid by workers. Suppose a colony has 20 unrelated queens with equal-sized broods, each singly mated, $F_{q_{\mu \mu}}=1$, and that there is no inbreeding, $F_{\phi \mu}=0$. Then $p=1 / 20, B_{w \rightarrow m}=$ $1 / 80$, and $B_{w \rightarrow f}=3 / 80$, so the ratio of the $B$ 's under worker control is still $1: 3$, independent of queen number.

Estimates for the interactions among relatives terms can also be obtained from the mating swarm data, and the assumption that the population is in sex allocation equilibrium. For queen control $(c=q)$, the LMC term, $F_{q \rightarrow m \rightarrow \mu}$, is equal to $F_{\mu \mu}$, since the haploid males each represent a gamete from a queen, and there are expected to be an equal number of males from all queens (by the $\mathrm{CMH}$ ); therefore the correlation between males is the correlation between a gamete from control and a gamete from a randomly chosen male with which a queen's son competes. If we continue to assume that queens contribute an equal number of males to the present mating swarm, as expected at equilibrium, then under queen control the extra value of a female for mating with related males, $F_{q \rightarrow f \rightarrow \mu}$ is again equal to $F_{\mu \mu}$. Similarly, the LRC term, $2 F_{q \rightarrow f \rightarrow \phi}+$ $F_{q \rightarrow f \rightarrow \phi \rightarrow \mu}$ is equal to $2 F_{\phi \mu}+F_{\mu \mu}$, the average number of $\mathrm{ibd}$ alleles from a queen per diploid female or haploid male in the mating swarm, as sampled by a randomly chosen queen's gamete, or equivalently, a male $(\mu)$ in the mating swarm. This term must be weighted by the probability that females from the same mating swarm will compete for resources. If a fraction $d$ of the mated females disperse before settling, then the probability of females from the same mating swarm competing is $(1-d)^{2}$ (Taylor 1987; Frank 1986b). The estimates for worker control can be derived in a similar manner.

The case of monogyny, single mating, no LRC, and no relatedness among queens has been studied for queen control (Hamilton 1979; Taylor and Bulmer 1980; Uyenoyama and Bengtsson 1982) and for worker control (Uyenoyama and Bengtsson 1982). If $N$ colonies contribute to the swarm, and each colony has sufficient resources to produce the equilibrium number of males, then by the $\mathrm{CMH}$ each will produce exactly the same number of males. So the amount of sib-mating is $1 / N$, independent of variance among colony output, and from 
Uyenoyama and Bengtsson (1982), the equilibrium proportion of male investment (males/(males + females)), $x^{*}$, is

(queen control) $\quad x^{*}=\frac{1}{2}\left(\frac{4 N-2}{4 N-1}\right)\left(\frac{N-1}{N}\right)$

(worker control) $x^{*}=\frac{N-1}{4 N-1}$.

These expressions can also be obtained from the above expressions for the $B$ 's and $F$ s, and Eq. (A.1). First, for the $B$ 's, set the probability of workers and offspring in the same colony having the same mother, $p$, to one, since monogyny is assumed; set the gametic correlation among mates of the same queen, $F_{q_{\mu \mu}}$, to one, since single mating is assumed; and set the correlation of uniting gametes, $F_{\varphi \mu}$, to $1 /(4 N-3)$, since the amount of sib-mating is $1 / N$ ( $\mathrm{Li} \mathrm{1976,} \mathrm{p.} \mathrm{244).} \mathrm{The} \mathrm{LMC}$ term for queen control, $F_{q \rightarrow m \rightarrow \mu}$ is equal to $F_{\mu \mu}$ (see above), the correlation among males in the mating swarm. Since queens are unrelated by assumption, males are related only when sibs, which occurs with probability $1 / N$, so $F_{\mu \mu}$ is $(1 / 2 N)\left(1+F_{\phi \mu}\right)$. Likewise, the queens' relatedness to females' mates, $F_{q \rightarrow f \rightarrow \mu}$, is also $F_{\mu \mu}$, as explained above. There is no LRC by assumption. Substituting these expressions into Eq. (A.1), and rearranging to give males/(males + females), yields Eq. (A. 2).

The solution for worker control, Eq. (A.3), is obtained by noting that the LMC term and the additional value for sisters' mates each depend on the probability of a sibling encountering a brother, $1 / N$, multiplied by a worker's number of ibd alleles in a haploid brother, $(1 / 4)\left(1+3 F_{\phi \mu}\right)$. Substituting into (A.1) yields Eq. (A.3).

\section{References}

Alexander RD, Sherman PW (1977) Local mate competition and parental investment in social insects. Science $196: 494-500$

Clark AB (1978) Sex ratio and local resource competition in a prosimian primate. Science $201: 163-165$

Fisher RA (1958) The genetical theory of natural selection, 2nd ed. Dover Press, New York

Frank SA (1985) Hierarchical selection theory and sex ratios. II. On applying the theory, and a test with fig wasps. Evolution 39:949-964

Frank SA (1986a) Hierarchical selection theory and sex ratios. I. General solutions for structured populations. Theor Pop Biol 29:312-342
Frank SA (1986b) The genetic value of sons and daughters. Heredity $56: 351-354$

Frank SA (1986c) Dispersal polymorphisms in subdivided populations. J Theor Biol 122:303-309

Frank SA (1987) Individual and population sex allocation patterns. Theor Popul Biol 31:47-74

Hamilton WD (1967) Extraordinary sex ratios. Science $156: 477-488$

Hamilton WD (1979) Wingless and fighting males in fig wasps in other insects. In: Blum MS, Blum NA (eds) Reproductive competition and sexual selection in insects. Academic, New York (pp 167-220)

Herbers JM (1984) Queen-worker conflict and eusocial evolution in a polygynous ant species. Evolution 38:631-643

Li CC (1976) A first course in population genetics. Boxwood, Pacific Grove, California

Nonacs P (1986) Ant reproductive stretegies and sex allocation theory. Q Rev Biol 61:1-21

Noonan KM (1979) Individual strategies of inclusive-fitnessmaximizing in the social wasp Polistes fuscatus (Hymenoptera:Vespidae). Ph.D. thesis, University of Michigan, Ann Arbor

Pamilo P, Crozier RH (1982) Measuring genetic relatedness in natural populations: methodology. Theor Popul Biol $21: 171-193$

Strassmann JE (1984) Female-biased sex ratios in social insects lacking morphological castes. Evolution 38:256-266

Taylor PD (1987) A first-order equivalence of genetic and inclusive fitness models. Theor Popul Biol (in press)

Taylor PD, Bulmer MG (1980) Local mate competition and the sex ratio. J Theor Biol 86:409-419

Trivers RL, Hare H (1976) Haplodiploidy and the evolution of social insects. Science 191:249-263

Uyenoyama MK, Bengtsson BO (1981) Towards a genetic theory for the evolution of the sex ratio. II. Haplodiploid and diploid models with sibling and parental control of the brood sex ratio and brood size. Theor Popul Biol 20:57-79

Uyenoyama MK, Bengtsson BO (1982) Towards a genetic theory for the evolution of the sex ratio. III. Parental and sibling control of brood investment ratio under partial sibmating. Theor Popul Biol 22:43-68

Wright S (1969) Evolution and the genetics of populations, Vol 2., University of Chicago Press, Chicago

Yamaguchi Y (1985) Sex ratios of an aphid subject to local mate competition with variable maternal condition. Nature $318: 460-462$ 